\title{
Fusarium chlamydosporum
}

National Cancer Institute

\section{Source}

National Cancer Institute. Fusarium chlamydosporum. NCI Thesaurus. Code C127711.

A species of filamentous fungi in the phylum Ascomycota. Infection with $\mathrm{F}$.

chlamydosporum is associated with superficial mycoses and keratitis in

immunocompetent individuals and with invasive and disseminated infection in

immunocompromised individuals. 